PROCEEDINGS OF THE

AMERICAN MATHEMATICAL SOCIETY

Volume 130, Number 5, Pages 1507-1513

S 0002-9939(01)06419-X

Article electronically published on December 20, 2001

\title{
NORMAL CHARACTERISTIC NUMBERS
}

\author{
R. E. STONG \\ (Communicated by Ralph Cohen)
}

\begin{abstract}
This paper computes the greatest common divisor of certain normal Pontrjagin numbers and Chern numbers. These calculations are of interest in studying singularities.
\end{abstract}

\section{INTRODUCTION}

Let $M^{4 n}$ be a closed smooth manifold of dimension $4 n$, and let $\bar{\wp}$ denote the Pontrjagin class of the stable normal bundle of $M$. One then obtains an integer $\bar{\wp}_{n}\left[M^{4 n}\right]$ by evaluation of the top dimensional Pontrjagin class on the fundamental homology class of $M$. The goal of this paper is to prove the following result.

Proposition. The greatest common divisor of the integers $\bar{\wp}_{n}\left[M^{4 n}\right]$ is $3^{k}$ where $k$ is the smallest integer $j$ for which $\alpha_{3}(2 n+j) \leq 3 j$.

For a prime $p, \alpha_{p}(x)$ is the sum of the digits in the $p$-adic expansion of $x$, or equivalently the minimum number of powers of $p$ needed to express $x$.

That $\sigma_{n}=\operatorname{gcd}\left\{\bar{\wp}_{n}\left[M^{4 n}\right]\right\}$ is of interest follows from the work of András Szücs 2]. He shows that the number of cusps of any stable smooth map of $M^{4 n}$ in $R^{6 n-1}$ is $\bar{\wp}_{n}\left[M^{4 n}\right]$, so the possible numbers of cusps is the group $\sigma_{n} Z$. He also describes (2], Remark 5) the method for calculating these numbers.

In an analogous way, one has

Proposition. The greatest common divisor of the normal Chern numbers $\bar{c}_{n}\left[M^{2 n}\right]$ of stably almost complex manifolds of dimension $2 n$ is $2^{k}$ where $k$ is the smallest integer $j$ for which $\alpha_{2}(n+j) \leq 2 j$.

Szücs has indicated that these numbers give the number of singular points for appropriate complex maps.

The general background information needed about characteristic classes may be found in [1].

\section{THE METHOD}

The function assigning to $M^{4 n}$ the value $\bar{\wp}_{n}\left[M^{4 n}\right]$ defines a ring homomorphism $\phi: \Omega_{*}^{S O} \rightarrow Z$ from the oriented cobordism ring to the integers. The torsion subgroup must go to zero under the homomorphism, so this gives $\phi: \Omega_{*}^{S O} /$ Tor $\rightarrow Z$. The ring $\Omega_{*}^{S O} /$ Tor is a polynomial ring $Z\left[x_{4 n}\right]$ on generators of dimension $4 n$.

Received by the editors September 7, 2000.

2000 Mathematics Subject Classification. Primary 57R20.

(C)2001 American Mathematical Society 
(Similarly, sending $M^{2 n}$ to $\bar{c}_{n}\left[M^{2 n}\right]$ gives a ring homomorphism $\phi: \Omega_{*}^{U} \rightarrow Z$ from complex cobordism to the integers, and $\Omega_{*}^{U}$ is a polynomial $\operatorname{ring} Z\left[x_{2 n}\right]$.)

The generator $x_{4}$ may be taken to be the class of the complex projective plane $\mathbb{C} P^{2}$, for which $\bar{\wp}_{1}\left(\mathbb{C} P^{2}\right)=-3$. Then $\phi(\underbrace{\mathbb{C} P^{2} \times \cdots \times \mathbb{C} P^{2}}_{n})=(-1)^{n} 3^{n}$, so $\sigma_{n}$ must be a power of 3 . (Similarly $x_{2}$ may be taken to be the class of $\mathbb{C} P^{1}$, with $\bar{c}_{1}\left[\mathbb{C} P^{1}\right]=-2$, so the greatest common divisor must be a power of 2.)

Because the problem is 3-primary, it suffices to consider 3-primary generators for $\Omega_{*}^{S O} /$ Tor and these generators are characterized by the fact that $s_{n}(\wp)\left[x_{4 n}\right]$ is nonzero mod 3 if $2 n+1$ is not a power of 3 and is divisible by 3 but not by 9 if $2 n+1$ is a power of 3 . For such 3 -primary indecomposables one computes the values of $\bar{\wp}_{n}\left[x_{4 n}\right]$, and $\sigma_{n}$ will be the greatest common divisor of the values $\bar{\wp}_{n}\left[x_{4 i_{1}} \cdots x_{4 i_{r}}\right]=\bar{\wp}_{i_{1}}\left[x_{4 i_{1}}\right] \cdots \bar{\wp}_{i_{r}}\left[x_{4 i_{r}}\right]$ for $i_{1}+\cdots+i_{r}=n$. Here $s_{n}(\wp)$ is the primitive class with $s_{n}(\wp)=\sum y_{i}^{2 n}$ if the Pontrjagin class has the form $\wp=\pi\left(1+y_{i}^{2}\right)$ with $\operatorname{dim} y_{i}=2$. (Similarly, 2-primary generators for $\Omega_{*}^{U}$ are characterized by having $s_{n}(c)\left[x_{2 n}\right]$ nonzero $\bmod 2$ if $n+1$ is not a power of 2 and being $2 \bmod 4$ if $n+1$ is a power of 2 . Here $s_{n}(c)=\sum y_{i}^{n}$ if $c=\pi\left(1+y_{i}\right)$ with $\operatorname{dim} y_{i}=2$.)

The remainder of the paper is devoted to finding appropriate generators and then finding the greatest common divisor for monomials in these generators.

\section{Generators}

This section will establish the following two results.

Lemma. There are 3-primary generators $x_{4 n}$ for $\Omega_{*}^{S O} /$ Tor for which the power of 3 dividing $\bar{\wp}_{n}\left[x_{4 n}\right]$ is

$$
\begin{cases}1 & \text { if } 2 n+1 \text { is a power of } 3, \\ \frac{1}{2}\left\{\alpha_{3}(2 n+1)-1\right\} & \text { if } 2 n+1 \text { is not a power of } 3 .\end{cases}
$$

Lemma. There are 2-primary generators $x_{2 n}$ for $\Omega_{*}^{U}$ for which the power of 2 dividing $\bar{c}_{n}\left[x_{2 n}\right]$ is

$$
\begin{cases}1 & \text { if } n+1 \text { is a power of } 2, \\ \alpha_{2}(n+1)-1 & \text { if } n+1 \text { is not a power of } 2 .\end{cases}
$$

Notice that for $x_{4}, 2 n+1=3$ and $\bar{\wp}_{1}\left[\mathbb{C} P^{2}\right]=-3$, and for $x_{2}, n+1=2$ and $\bar{c}_{1}\left[x_{2}\right]=-2$. Thus, one has the result for $n=1$, and more importantly, for $2 n+1=3^{1}, n+1=2^{1}$.

The easiest case is for $n+1$ not divisible by 2 and $2 n+1$ not divisible by 3 . For this consider the manifold $\mathbb{C} P^{n}$. The cohomology of $\mathbb{C} P^{n}$ is $Z[\alpha] /\left(\alpha^{n+1}=0\right)$ and the Chern class is $c=(1+\alpha)^{n+1}$. Then $s_{n}(c)=(n+1) \alpha^{n}$, so $s_{n}(c)\left[\mathbb{C} P^{n}\right]=n+1$ which is not divisible by 2 if $n+1$ is not divisible by 2 . Then $\bar{c}=\frac{1}{(1+\alpha)^{n+1}}$ and $\bar{c}_{n}\left[\mathbb{C} P^{n}\right]$ is the coefficient of $\alpha^{n}$ in

$$
\frac{1}{(1+\alpha)^{n+1}}=\sum_{i=0}^{\infty}\left(\begin{array}{c}
n+i \\
i
\end{array}\right)(-\alpha)^{i}
$$

which is $(-1)^{n}\left(\begin{array}{c}2 n \\ n\end{array}\right)$. 
Now, it is well known that the power of $p$ dividing $x !$ is $\left(x-\alpha_{p}(x)\right) /(p-1)$, so the power of $p$ dividing the binomial coefficient $\left(\begin{array}{l}x \\ y\end{array}\right)=\frac{x !}{y !(x-y) !}$ is

$$
\begin{gathered}
\frac{1}{(p-1)}\left\{x-\alpha_{p}(x)-\left(y-\alpha_{p}(y)\right)-\left(x-y-\alpha_{p}(x-y)\right)\right\} \\
=\frac{1}{(p-1)}\left\{\alpha_{p}(y)+\alpha_{p}(x-y)-\alpha_{p}(x)\right\} .
\end{gathered}
$$

The power of 2 dividing $\bar{c}_{n}\left[\mathbb{C} P^{n}\right]=(-1)^{n}\left(\begin{array}{c}2 n \\ n\end{array}\right)$ is then $2 \alpha_{2}(n)-\alpha_{2}(2 n)=\alpha_{2}(n)$ since $\alpha_{2}(2 n)=\alpha_{2}(n)$. Since $n+1$ is odd, $n$ is even and $\alpha_{2}(n+1)=\alpha_{2}(n)+1$ or $\alpha_{2}(n)=\alpha_{2}(n+1)-1$, as in the lemma.

For $\mathbb{C} P^{2 n}$, the Chern class is $c=(1+\alpha)^{2 n+1}$ and the Pontrjagin class is $\wp=$ $\left(1+\alpha^{2}\right)^{2 n+1}$. Then $s_{n}(\wp)=s_{2 n}(c)=(2 n+1) \alpha^{2 n}$ so $s_{n}(\wp)\left[\mathbb{C} P^{2 n}\right]=2 n+1$ which is not divisible by 3 if $2 n+1$ is not divisible by 3 . Then $\bar{\wp}_{n}\left[\mathbb{C} P^{2 n}\right]$ is the coefficient of $\alpha^{2 n}$ in

$$
\bar{\wp}=\frac{1}{\left(1+\alpha^{2}\right)^{2 n+1}}=\sum_{i=0}^{\infty}\left(\begin{array}{c}
2 n+i \\
i
\end{array}\right)\left(-\alpha^{2}\right)^{i},
$$

which is $(-1)^{n}\left(\begin{array}{c}2 n+n \\ n\end{array}\right)=(-1)^{n}\left(\begin{array}{c}3 n \\ n\end{array}\right)$. The power of 3 dividing $\left(\begin{array}{c}3 n \\ n\end{array}\right)$ is

$$
\frac{1}{2}\left\{\alpha_{3}(n)+\alpha_{3}(2 n)-\alpha_{3}(3 n)\right\}=\frac{1}{2} \alpha_{3}(2 n)
$$

since $\alpha_{3}(3 n)=\alpha_{3}(n)$. Since $2 n+1$ is not divisible by $3, \alpha_{3}(2 n+1)=\alpha_{3}(2 n)+1$, so $\frac{1}{2} \alpha_{3}(2 n)=\frac{1}{2}\left\{\alpha_{3}(2 n+1)-1\right\}$, as in the lemma.

If $2 n+1$ is divisible by 3 , but is not a power of 3 , then $2 n+1=a_{1} \cdot 3+a_{2} \cdot 3^{2}+$ $\cdots+a_{r} \cdot 3^{r}$ is not just a single power of 3 . Because $2 n+1$ is odd and 3 is odd, at least one of the coefficients $a_{i}$ must be odd, hence is 1 , so fix $i$ with $a_{i}=1$, and let $m=2 n+1-3^{i}$ (nonzero since $2 n+1 \neq 3^{i}$ ).

Let $M^{4 n} \subset \mathbb{C} P^{3^{i}} \times \mathbb{C} P^{m}$ be the submanifold dual to $\alpha+\beta$ where $H^{*}\left(\mathbb{C} P^{3^{i}}\right)=$ $Z[\alpha] /\left(\alpha^{3^{i}+1}=0\right)$ and $H^{*}\left(\mathbb{C} P^{m}\right)=Z[\beta] /\left(\beta^{m+1}=0\right)$. The Chern class of $M$ is $\frac{(1+\alpha)^{3^{i}+1}(1+\beta)^{m+1}}{1+\alpha+\beta}$ and the $s$-class is

$$
s_{n}(\wp)=s_{2 n}(c)=\left(3^{i}+1\right) \alpha^{2 n}+(m+1) \beta^{2 n}-(\alpha+\beta)^{2 n}=-(\alpha+\beta)^{2 n},
$$

with $s_{n}(\wp)[M]=-(\alpha+\beta)^{2 n}[M]=-(\alpha+\beta)^{2 n+1}\left[\mathbb{C} P^{3^{i}} \times \mathbb{C} P^{m}\right]$, which is the coefficient of $\alpha^{3^{i}} \beta^{m}$ in $-(\alpha+\beta)^{2 n+1}$. This is $-\left(\begin{array}{c}2 n+1 \\ 3^{i}\end{array}\right)$ and is nonzero mod 3 . Thus $M^{4 n}$ is a 3 -primary generator for $\Omega_{*}^{S O} /$ Tor.

The Pontrjagin class of $M$ is $\wp=\frac{\left(1+\alpha^{2}\right)^{3^{i}+1}\left(1+\beta^{2}\right)^{m+1}}{1+(\alpha+\beta)^{2}}$, so

$$
\begin{aligned}
\bar{\wp}[M] & =\bar{\wp}[M]=\frac{1+(\alpha+\beta)^{2}}{\left(1+\alpha^{2}\right)^{3^{i}+1}\left(1+\beta^{2}\right)^{m+1}}[M] \\
& =\frac{\left(1+(\alpha+\beta)^{2}\right)(\alpha+\beta)}{\left(1+\alpha^{2}\right)^{3^{i}+1}\left(1+\beta^{2}\right)^{m+1}}\left[\mathbb{C} P^{3^{i}} \times \mathbb{C} P^{m}\right],
\end{aligned}
$$

which is the coefficient of $\alpha^{3^{i}} \beta^{m}$ in

$$
\frac{\left\{\alpha+\beta+\alpha^{3}+3 \alpha^{2} \beta+3 \alpha \beta^{2}+\beta^{3}\right\}}{\left(1+\alpha^{2}\right)^{3^{i}+1}\left(1+\beta^{2}\right)^{m+1}} .
$$


Since $3^{i}$ is odd and $m$ is even, only terms $\alpha^{\text {odd }} \beta^{\text {even }}$ in the numerator can contribute to $\alpha^{3^{i}} \beta^{m}$ and this is the coefficient of $\alpha^{3^{i}} \beta^{m}$ in

$$
\begin{aligned}
& \left\{\alpha+\alpha^{3}+3 \alpha \beta^{2}\right\}\left(\sum_{j=0}^{\infty}\left(\begin{array}{c}
3^{i}+j \\
j
\end{array}\right)\left(-\alpha^{2}\right)^{j}\right)\left(\sum_{\ell=0}^{\infty}\left(\begin{array}{c}
m+\ell \\
\ell
\end{array}\right)\left(-\beta^{2}\right)^{\ell}\right) \\
& =(-1)^{n}\left(\begin{array}{c}
3^{i}+\frac{3^{i}-1}{2} \\
\frac{3^{i}-1}{2}
\end{array}\right)\left(\begin{array}{c}
m+\frac{m}{2} \\
\frac{m}{2}
\end{array}\right)+(-1)^{n-1}\left(\begin{array}{c}
3^{i}+\frac{3^{i}-1}{2}-1 \\
\frac{3^{i}-1}{2}-1
\end{array}\right)\left(\begin{array}{c}
m+\frac{m}{2} \\
\frac{m}{2}
\end{array}\right) \\
& +(-1)^{n-1} 3\left(\begin{array}{c}
3^{i}+\frac{3^{i}-1}{2} \\
\frac{3^{i}-1}{2}
\end{array}\right)\left(\begin{array}{c}
m+\frac{m}{2}-1 \\
\frac{m}{2}-1
\end{array}\right) .
\end{aligned}
$$

Now

$$
\left(\begin{array}{c}
m+\frac{m}{2} \\
\frac{m}{2}
\end{array}\right)=\left(\begin{array}{c}
3\left(\frac{m}{2}\right) \\
\frac{m}{2}
\end{array}\right)=\frac{3\left(\frac{m}{2}\right)}{\left(\frac{m}{2}\right)}\left(\begin{array}{c}
3\left(\frac{m}{2}\right)-1 \\
\frac{m}{2}-1
\end{array}\right)=3\left(\begin{array}{c}
m+\frac{m}{2}-1 \\
\frac{m}{2}-1
\end{array}\right),
$$

so the first and last terms cancel, and

$$
\left(\begin{array}{c}
3^{i}+a \\
a
\end{array}\right) \not \equiv 0 \bmod 3 \text { if } a<3^{i}
$$

Thus the power of 3 dividing $\bar{\wp}_{n}[M]$ is the power of 3 dividing $\left(\begin{array}{c}m+\frac{m}{2} \\ \frac{m}{2}\end{array}\right)=$ $\left(\begin{array}{c}3\left(\frac{m}{2}\right) \\ \frac{m}{2}\end{array}\right)$ which is $\frac{1}{2}\left\{\alpha_{3}\left(\frac{m}{2}\right)+\alpha_{3}(m)-\alpha_{3}\left(\frac{3 m}{2}\right)\right\}=\frac{1}{2} \alpha_{3}(m)$, since $\alpha_{3}\left(\frac{m}{2}\right)=$ $\alpha_{3}\left(\frac{3 m}{2}\right)$. Now $2 n+1=m+3^{i}$, with $3^{i}$ not appearing in the 3 -adic expansion of $m$, so $\alpha_{3}(2 n+1)=\alpha_{3}(m)+1$ and $\frac{1}{2} \alpha_{3}(m)=\frac{1}{2}\left\{\alpha_{3}(2 n+1)-1\right\}$, as in the lemma.

If $n+1$ is divisible by 2 , but is not a power of 2 , one may choose $i$ for which $2^{i}$ appears with coefficient 1 in the 2-adic expansion of $n+1$ and let $m=n+1-2^{i}$. (For example, $n+1=2^{i}(2 v+1)$ and one may let $m=2^{i+1} v$.)

Let $M^{2 n} \subset \mathbb{C} P^{2^{i}} \times \mathbb{C} P^{m}$ be the submanifold dual to $\alpha+\beta$. The Chern class of $M$ is $\frac{(1+\alpha)^{2^{i}+1}(1+\beta)^{m+1}}{1+\alpha+\beta}$ and the $s$-class is $s_{n}(c)=\left(2^{i}+1\right) \alpha^{n}+(m+1) \beta^{n}-(\alpha+\beta)^{n}=$ $-(\alpha+\beta)^{n}$, so $s_{n}(c)[M]=-(\alpha+\beta)^{n}[M]=-(\alpha+\beta)^{n+1}\left[\mathbb{C} P^{2^{i}} \times \mathbb{C} P^{m}\right]=-\left(\begin{array}{c}n+1 \\ 2^{i}\end{array}\right)$, which is odd. Also

$$
\begin{aligned}
\bar{c}_{n}[M] & =\bar{c}[M]=\frac{(1+\alpha+\beta)}{(1+\alpha)^{2^{i}+1}(1+\beta)^{m+1}}[M] \\
& =\frac{(1+\alpha+\beta)(\alpha+\beta)}{(1+\alpha)^{2^{i}+1}(1+\beta)^{m+1}}\left[\mathbb{C} P^{2^{i}} \times \mathbb{C} P^{m}\right],
\end{aligned}
$$

which is the coefficient of $\alpha^{2^{i}} \beta^{m}$ in

$$
\left(\alpha+\beta+\alpha^{2}+2 \alpha \beta+\beta^{2}\right)\left(\sum_{j=0}^{\infty}\left(\begin{array}{c}
2^{i}+j \\
j
\end{array}\right)(-\alpha)^{j}\right)\left(\sum_{\ell=0}^{\infty}\left(\begin{array}{c}
m+\ell \\
\ell
\end{array}\right)(-\beta)^{\ell}\right)
$$


which is

$$
\begin{aligned}
(-1)^{n} & \left(\begin{array}{c}
2^{i}+2^{i}-1 \\
2^{i}-1
\end{array}\right)\left(\begin{array}{c}
2 m \\
m
\end{array}\right) \\
& +(-1)^{n}\left(\begin{array}{c}
2^{i}+1 \\
2^{i}
\end{array}\right)\left(\begin{array}{c}
2 m-1 \\
m-1
\end{array}\right)+(-1)^{n-1}\left(\begin{array}{c}
2^{i}+2^{i}-2 \\
2^{i}-2
\end{array}\right)\left(\begin{array}{c}
2 m \\
m
\end{array}\right) \\
& +(-1)^{n-1} 2\left(\begin{array}{c}
2^{i}+2^{i}-1 \\
2^{i}-1
\end{array}\right)\left(\begin{array}{c}
2 m-1 \\
m-1
\end{array}\right)+(-1)^{n-1}\left(\begin{array}{c}
2^{i+1} \\
2^{i}
\end{array}\right)\left(\begin{array}{c}
2 m-2 \\
m-2
\end{array}\right) \\
= & (-1)^{n}\left(\begin{array}{c}
2^{i+1}-1 \\
2^{i}-1
\end{array}\right)\left(\begin{array}{c}
2 m-1 \\
m-1
\end{array}\right) \\
& \times\left[\frac{2 m}{m}+\frac{2^{i+1}}{2^{i}}-\frac{2^{i}-1}{2^{i+1}-1} \cdot \frac{2 m}{m}-2-\frac{2^{i+1}}{2^{i}} \cdot \frac{m-1}{2 m-1}\right] .
\end{aligned}
$$

The sum is

$$
\begin{aligned}
2+2-\frac{2^{i+1}-2}{2^{i+1}-1}-2-\frac{2 m-2}{2 m-1} & =2-\left(1-\frac{1}{2^{i+1}-1}\right)-\left(1-\frac{1}{2 m-1}\right) \\
& =\frac{1}{2^{i+1}-1}+\frac{1}{2 m-1}=\frac{2 m+2^{i+1}-2}{\left(2^{i+1}-1\right)(2 m-1)} \\
& =\frac{2(n+1)-2}{\left(2^{i+1}-1\right)(2 m-1)}
\end{aligned}
$$

so

$$
\begin{aligned}
\bar{c}_{n}[M] & =(-1)^{n}\left(\begin{array}{c}
2^{i+1}-1 \\
2^{i}-1
\end{array}\right)\left(\begin{array}{c}
2 m-1 \\
m-1
\end{array}\right) 2 \frac{n}{\left(2^{i+1}-1\right)(2 m-1)} \\
& =(-1)^{n}\left(\begin{array}{c}
2^{i+1}-1 \\
2^{i}-1
\end{array}\right)\left(\begin{array}{c}
2 m \\
m
\end{array}\right) \frac{n}{\left(2^{i+1}-1\right)(2 m-1)} .
\end{aligned}
$$

Since $\left(\begin{array}{c}2^{i+1}-1 \\ 2^{i}-1\end{array}\right), n, 2^{i+1}-1$, and $2 m-1$ are all odd, the power of 2 dividing $\bar{c}_{n}[M]$ is the power of 2 dividing $\left(\begin{array}{c}2 m \\ m\end{array}\right)$ which is $\alpha_{2}(m)$. Since $n+1=m+2^{i}, \alpha_{2}(m)=$ $\alpha_{2}(n+1)-1$, as in the lemma.

For $2 n+1=3^{r}$ with $r>1$, let $M^{4 n} \subset \mathbb{C} P^{3^{r}}$ be the degree 3 hypersurface (submanifold dual to $3 \alpha$ ). The Chern class of $M$ is $\frac{(1+\alpha)^{3^{r}+1}}{1+3 \alpha}$ so $s_{n}(\wp)[M]=$ $s_{2 n}(c)[M]=\left\{\left(3^{r}+1\right) \alpha^{2 n}-(3 \alpha)^{2 n}\right\}[M]=\left\{\left(3^{r}+1\right) \alpha^{2 n}-(3 \alpha)^{2 n}\right\} 3 \alpha\left[\mathbb{C} P^{2 n+1}\right] \equiv$ $-3 \alpha^{2 n+1}\left[\mathbb{C} P^{2 n+1}\right] \equiv-3 \bmod 9$. Thus $M$ is a 3 -primary generator of $\Omega_{*}^{S O} /$ Tor. The Pontrjagin class of $M$ is $\frac{\left(1+\alpha^{2}\right)^{3^{r}+1}}{1+(e \alpha)^{2}}$, so $\bar{\wp}_{n}[M]$ is the coefficient of $\alpha^{3^{r}}$ in

$$
\frac{1+(3 \alpha)^{2}}{\left(1+\alpha^{2}\right)^{3^{r}+1}}(3 \alpha)=\left(3 \alpha+27 \alpha^{3}\right)\left(\sum_{k=0}^{\infty}\left(\begin{array}{c}
3^{r}+k \\
k
\end{array}\right)\left(-\alpha^{2}\right)^{k}\right)
$$


which is

$$
3\left(\begin{array}{c}
3^{r}+\frac{3^{r}-1}{2} \\
\frac{3^{r}-1}{2}
\end{array}\right)(-1)^{\frac{3^{r}-1}{2}}+27\left(\begin{array}{c}
3^{r}+\frac{3^{r}-3}{2} \\
\frac{3^{r}-3^{2}}{2}
\end{array}\right)(-1)^{\frac{3^{r}-3}{2}} \text { and }\left(\begin{array}{c}
3^{r}+\frac{3^{r}-1}{2} \\
\frac{3^{r}-1}{2}
\end{array}\right) \not \equiv 0,
$$

modulo 3 , so this is divisible by 3 , but not by 9 . Thus the power of 3 dividing $\bar{\wp}_{n}[M]$ is 1 , as in the lemma.

For $n+1=2^{r}$ with $r>1$, let $M^{2 n} \subset \mathbb{C} P^{2^{r}}$ be the degree 2 hypersurface (submanifold dual to $2 \alpha$ ). The Chern class of $M$ is $\frac{(1+\alpha)^{2^{r}+1}}{1+2 \alpha}$ so

$$
s_{n}(c)[M]=\left\{\left(2^{r}+1\right) \alpha^{n}-(2 \alpha)^{n}\right\}[M]=\left\{\left(2^{r}+1\right) \alpha^{n}-(2 \alpha)^{n}\right\}(2 \alpha)\left[\mathbb{C} P^{2 n+1}\right],
$$

and this is $2 \bmod 4$. Thus $M$ is a 2-primary generator of $\Omega_{*}^{U}$. Then $\bar{c}_{n}[M]$ is the coefficient of $\alpha^{n+1}$ in

$$
\frac{1+2 \alpha}{(1+\alpha)^{2^{r}+1}} \cdot(2 \alpha)=\left(2 \alpha+4 \alpha^{2}\right)\left(\sum_{j=0}^{\infty}\left(\begin{array}{c}
2^{r}+j \\
j
\end{array}\right)(-\alpha)^{j}\right),
$$

which is $-2\left(\begin{array}{c}2^{r+1}-1 \\ 2^{r}-1\end{array}\right)+4\left(\begin{array}{c}2^{r+1}-2 \\ 2^{r}-2\end{array}\right) \equiv 2 \bmod 4$ since both binomial coefficients are odd. Thus the power of 2 dividing $\bar{c}_{n}[M]$ is 1 , as in the lemma.

\section{THE GREATEST COMMON DIVISOR}

The argument for finding the greatest common divisor is essentially the same in the two cases, so the proof will be given for Pontrjagin classes.

Let $k_{n}=\min \left\{j \mid \alpha_{3}(2 n+j) \leq 3 j\right\}$ and

$$
3^{g_{n}}=\operatorname{gcd}\left\{\bar{\wp}_{n_{1}}\left[x_{4 n_{1}}\right] \cdots \bar{\wp}_{n_{r}}\left[x_{4 n_{r}}\right] \mid n_{1}+\cdots+n_{r}=n\right\} .
$$

One has certain easy facts.

Fact. The power of 3 dividing $\bar{\wp}_{n}\left[x_{4 n}\right]$ is 1 if and only if $2 n+1$ can be written as the sum of three powers of 3 .

If $2 n+1=3^{r}$, then $2 n+1=3^{r-1}+3^{r-1}+3^{r-1}$, and if $\frac{1}{2}\left\{\alpha_{3}(2 n+1)-1\right\}=1$, then $\alpha_{3}(2 n+1)=3$, so $2 n+1$ is a sum of 3 powers of 3 .

Fact. $\alpha_{3}(x) \equiv x \bmod 2$.

Fact. $\alpha_{3}(2 n+n) \leq 3 n$, so $k_{n} \leq n$.

Obviously, $3 n=2 n+n$ is the sum of $3 n$ ones.

Fact. If $\alpha_{3}(2 n+j) \leq 3 j$, then $\alpha_{3}(2 n+(j+1)) \leq 3(j+1)$.

Since $\alpha_{3}(2 n+(j+1)) \leq \alpha_{3}(2 n+j)+1$, this is clear.

Fact. If $\alpha_{3}(2 n+j) \leq 3 j$ and $j \leq n$, then $2 n+j$ can be written as the sum of $3 j$ powers of 3 .

If $\alpha_{3}(2 n+j)=r \leq 3 j$, then $2 n+j$ is the sum of $r$ powers of 3 . Replacing $3^{x}$ by $3^{x-1}+3^{x-1}+3^{x-1}$ increases the number of powers of 3 by 2 , and one may write $2 n+j$ as the sum of any number of powers of 3 congruent to $r \bmod 2$ up to a maximum of $2 n+j$ powers of 3 (all 1's). Now $\alpha_{3}(2 n+j) \equiv 2 n+j \equiv r \equiv j \equiv 3 j$ mod 2 and $3 j \leq 2 n+j$, so $2 n+j$ can be written as a sum of $3 j$ powers of 3 . 
Corollary. If $\alpha_{3}(2 n+j) \leq 3 j$ with $j \leq n$, then $g_{n} \leq j$. Thus $g_{n} \leq k_{n}$.

Proof. If $\alpha_{3}(2 n+j) \leq 3 j$ and $j \leq n$, then $2 n+j$ is the sum of $3 j$ powers of 3 . One can then write $2 n+j=\left(2 n_{1}+1\right)+\cdots+\left(2 n_{j}+1\right)$ where each $2 n_{i}+1=3^{a_{i}}+3^{b_{i}}+3^{c_{i}}$ uses three of the powers of 3 . Then $2 n=2 n_{1}+\cdots+2 n_{j}$, and the power of 3 dividing $\bar{\wp}_{n_{1}}\left[x_{4 n_{1}}\right] \cdots \bar{\wp}_{n_{j}}\left[x_{4 n_{j}}\right]$ is $j$. Thus $g_{n} \leq j$.

Now suppose that $g_{m}=k_{m}$ for $m<n$, and that $g_{n}$ is the power of 3 dividing $\bar{\wp}_{n_{1}}\left[x_{4 n_{1}}\right] \cdots \bar{\wp}_{n_{r}}\left[x_{4 n_{r}}\right]$ with $2 n=2 n_{1}+\cdots+2 n_{r}$.

If $r=1$, then the power of 3 dividing $\bar{\wp}_{n}\left[x_{4 n}\right]$ is $g_{n}$. Then either $g_{n}=1(2 n+1=$ $\left.3^{j}\right)$ so $\alpha_{3}(2 n+1)=1 \leq 3 \cdot 1$ or $g_{n}=\frac{1}{2}\left\{\alpha_{3}(2 n+1)-1\right\}$ giving $\alpha_{3}(2 n+1)=1+2 g_{n}$ so $\alpha_{3}\left(2 n+g_{n}\right)=\alpha_{3}\left(2 n+1+\left(g_{n}-1\right)\right) \leq \alpha_{3}(2 n+1)+\left(g_{n}-1\right)=3 g_{n}$. Thus $\alpha_{3}\left(2 n+g_{n}\right) \leq 3 g_{n}$ and $k_{n} \leq g_{n}$.

If $r>1$ and $g_{n}$ is the power of 3 dividing $\bar{\wp}_{n_{1}}\left[x_{4 n_{1}}\right] \cdots \bar{\wp}_{n_{r}}\left[x_{4 n_{r}}\right]$, then the power of 3 dividing each $\bar{\wp}_{n_{i}}\left[x_{4 n_{i}}\right]$ must be $g_{n_{i}}$ (otherwise one can replace $x_{4 n_{i}}$ by a decomposable class to decrease the power of 3), and so $g_{n_{i}}=k_{n_{i}}$ inductively and $g_{n}=g_{n_{1}}+\cdots+g_{n_{r}}$. Thus $\alpha_{3}\left(2 n_{i}+g_{n_{i}}\right) \leq 3 g_{n_{i}}$ and $2 n+g_{n}=\left(2 n_{1}+g_{n_{1}}\right)+\cdots+$ $\left(2 n_{r}+g_{n_{r}}\right)$, so

$$
\alpha_{3}\left(2 n+g_{n}\right) \leq \alpha_{3}\left(2 n_{1}+g_{n_{1}}\right)+\cdots+\alpha_{3}\left(2 n_{r}+g_{n_{r}}\right) \leq 3 g_{n_{1}}+\cdots+3 g_{n_{r}}=3 g_{n} .
$$

Thus $k_{n} \leq g_{n}$.

Since $k_{n} \leq g_{n}$ and $g_{n} \leq k_{n}$, one obtains $g_{n}=k_{n}$ completing the induction and establishing the proposition.

\section{REFERENCES}

[1] J. W. Milnor and J. D. Stasheff, Characteristic classes, Ann. Math. Studies, No. 76, Princeton University Press, 1974. MR 55:13428

[2] A. Szücs, On the singularities of hyperplane projections of immersions, Bull. London Math. Soc. 32 (2000), 364-374. MR 2001c:57031

Department of Mathematics, University of Virginia, P.O. Box 400137, CharlottesVILLE, VIRGINIA 22904 